\title{
Influence of cognitive reserve on neuropsychological functioning in bipolar disorder: Findings from a 5-year longitudinal study
}

\author{
Kristin H Hinrichs $^{1}$ (D) | Rebecca E Easter ${ }^{1}$ | Kaley Angers ${ }^{1}$ | Bethany Pester ${ }^{1}$ | \\ Zongshan Lai $^{1}$ | David F Marshall ${ }^{1}$ | Masoud Kamali ${ }^{1,3}$ | Melvin Mclnnis ${ }^{1}$ | \\ Scott A Langenecker ${ }^{2}$ | Kelly A Ryan ${ }^{1}$
}

\footnotetext{
${ }^{1}$ Department of Psychiatry, University of Michigan, Ann Arbor, MI, USA

${ }^{2}$ Department of Psychiatry, University of Illinois at Chicago, Chicago, IL, USA

${ }^{3}$ Department of Psychiatry, Massachusetts General Hospital, Boston, MA, USA
}

Correspondence: Kelly Ryan, Department of Psychiatry, University of Michigan, Ann Arbor, MI, USA.

Email: karyan@med.umich.edu

\section{Funding information}

Heinz C. Prechter Bipolar Research Fund; University of Michigan Depression Center; Richard Tam Foundation.
Objectives: The present study examined the 5 -year longitudinal course of cognitive functioning in a large sample of well-characterized patients with bipolar disorder (BP), compared to healthy controls ( $\mathrm{HCs}$ ), and the influence of cognitive reserve factors (e.g., education and IQ) on cognitive change over time.

Methods: Participants included 159 individuals diagnosed with BP and $54 \mathrm{HCs}$ recruited as part of a longitudinal naturalistic study of BP who had completed neuropsychological testing at the time of their enrollment and again 5 years later.

Results: The overall relative rate of change did not differ between the BP and $\mathrm{HC}$ groups. In total, $46.5 \%$ of the BP group and $37 \%$ of the $\mathrm{HC}$ group showed evidence of decline on at least one measure over time. T-test analyses did not find differences between BP 'decliners' and 'non-decliners' in cognitive reserve variables. However, we found that higher baseline intellectual ability was associated with more stability in cognitive test scores over time for the BP group. Results of linear regression modeling revealed that lower verbal IQ and education were related to increased cognitive decline in specific domains in the BP group.

Conclusions: This study has explored the influence of cognitive reserve on preservation of specific cognitive abilities over time in BP. The BP group did not demonstrate accelerated cognitive decline over 5 years compared to the $\mathrm{HC}$ group. Although the trajectory of cognitive change over time was similar between BP patients and $\mathrm{HCs}$, higher overall intellectual ability may be a protective factor against cognitive decline, particularly for BP patients.

KEYWORDS

bipolar disorder, clinical neuropsychology, cognitive reserve

\section{1 | INTRODUCTION}

It has been well established that individuals with bipolar disorder (BP) typically achieve lower neuropsychological test scores compared to healthy controls ( $\mathrm{HCs}$ ) across a number of cognitive domains, including attention, memory, psychomotor speed, and executive functioning. ${ }^{1-4}$
However, few studies have examined if and how these cognitive deficits change over time. Past studies have found that older individuals with BP tend to have more functional impairment and neurocognitive impairment and higher rates of dementia. ${ }^{5-7}$ These findings lead to the assumption that individuals with BP may be at increased risk for cognitive decline and therefore suffer a progressive, accelerated 
neurodegeneration over time., ${ }^{8,9}$ Further, previous cross-sectional work by our group ${ }^{10}$ has demonstrated an additive effect of aging and cognitive burden on health-related quality of life, as well as evidence of an age by BP diagnosis interaction in terms of performance on emotion processing, processing speed, and executive functioning tasks. These findings highlight the importance of studying cognitive changes in aging individuals affected by BP.

Although relatively sparse, a number of recent articles have proposed that progression of cognitive symptoms over time may not differ between individuals diagnosed with $\mathrm{BP}$ and $\mathrm{HC}$ subjects. ${ }^{11-13}$ In a recent review article examining longitudinal and cross-sectional research on the trajectory of neuropsychological dysfunction among those with BP, Strejilevich and colleagues ${ }^{13}$ concluded that evidence does not support progressive neurocognitive decline over time. They noted, however, that many of the studies to date had methodological limitations such as small sample size and brief neuropsychological batteries. In other recent work, Gildengers and colleagues ${ }^{11}$ explored longitudinal neuropsychological performances in BP over 2 years and found that, although individuals with BP tended to score lower on cognitive measures overall, the change in scores did not differ compared to HCs, suggesting that BP was not related to an accelerated rate of cognitive decline as was previously assumed. Similarly, Santos and colleagues analyzed the longitudinal course of BP cognitive trajectory over a longer time period and again found that BP participants demonstrated a similar cognitive trajectory over time compared to controls, despite performing at lower levels on neuropsychological testing. Again, these findings suggest that having a BP diagnosis was not associated with progressive neurocognitive decline. Further, this work reported that the clinical symptom course of BP (measured by number of mood episodes) was unrelated to cognitive performance over time, indicating that cognitive deficits in BP tend to be stable, following a course similar to that in individuals without a significant psychiatric condition. Although compelling, data were obtained in a small sample size of 80 BP participants, and further exploration with larger samples and over longer periods of time is needed to better evaluate this hypothesis. As normative age-related changes can be notable and highly variable studies finding, a null effect of disease by age are likely underpowered with smaller samples over shorter periods of time. Overall, analysis of neurocognitive change in BP is a relatively new area of study, and most work done in the area has been cross-sectional in nature, utilizes small sample sizes over a short follow-up time interval, and has used a brief incomprehensive test battery.

While patterns of cognitive dysfunction across BP mood states have been found in executive functioning, psychomotor speed, attention, and memory, ${ }^{4}$ there is a large amount of heterogeneity in cognitive profiles and presentations, ${ }^{9}$ with patients ranging from no impairment to substantial impairment. ${ }^{14,15}$ Many individual differences in cognitive impairment lead to questions regarding possible environmental, experiential, or social factors that influence the development and change of neurocognitive performance over time. Cognitive reserve $(\mathrm{CR})$ is an important factor that could be contributing to this variability in neuropsychological performances in those with BP. The theory of $\mathrm{CR}$ asserts that some individuals have more resilient brains than others and can sustain higher levels of pathology or damage before exhibiting significant behavioral symptoms. This is thought to be due to accumulation of more efficient, larger, or stronger neural networks. ${ }^{16-19}$ Because CR cannot be easily quantified, proxy measures such as premorbid intellectual ability, education, occupational attainment, and leisure activity have been used as surrogate variables and are established as standards of CR measurement in the literature. ${ }^{20}$

CR has most frequently been adopted in research on acquired or neurodegenerative conditions, such as Alzheimer's disease, traumatic brain injury, and vascular disease ${ }^{21-28}$ however, CR has only recently been applied to neuropsychiatric disorders, such as schizophrenia, post-traumatic stress disorder, and BP, to help explain variability in symptom severity and neuropsychological correlates. ${ }^{1,29,30}$ In one of the few studies examining CR in BP, Forcada and colleagues ${ }^{31}$ found that BP participants with lower neuropsychological functioning (in verbal and visual memory and executive functioning) also tended to have lower psychosocial functioning, education, occupational attainment, and leisure activities compared to the control group. ${ }^{31}$ Similarly, in 2015, Anaya and colleagues ${ }^{32}$ studied the relationship between CR variables and BP neuropsychological functioning in a large sample of 200 participants. They reported that higher CR was indeed associated with better cognitive functioning in processing speed, working memory, verbal and visual memory, executive functioning, and attention. Therefore, preliminary support exists for the importance of premorbid characteristics in the severity of cognitive and functional impairment in $\mathrm{BP}^{33}$ although, to this point, no longitudinal study has explored how CR factors may influence change in neurocognitive ability over time with a longitudinal research design.

Clearly, examining potential CR effects of neuropsychiatric conditions is difficult, as the accumulation of CR is probably related to the clinical symptoms themselves. ${ }^{29}$ Clinical symptoms that are present at an earlier age and severe enough to interfere with daily functioning can significantly impact the patient's ability to complete advanced schooling, obtain steady high-level employment, or partake in other enriching experiences. ${ }^{34,35}$ While careful consideration of functional impairment should be taken, discovery of psychosocial moderators of neuropsychological change over time may have a significant impact on treatments and early interventions among individuals with BP symptoms. CR may be an important factor to consider, given its implications in other neurocognitive conditions. Detection of a CR effect on neurocognitive change over time may provide important insights into cognitive resiliency and how to achieve it.

The present study examined the 5-year longitudinal course of cognitive functioning in a large sample of well-characterized patients with $\mathrm{BP}$, and the influence of CR factors on any changes in cognitive functioning over time. First, we hypothesized that group differences would exist between groups in terms of neuropsychological test scores, consistent with previous literature. Secondly, based on some of the limited evidence, we expected that differences would not exist between BP patients and $\mathrm{HCs}$ in terms of change in neuropsychological test scores over 5 years, and that groups would thus show similar cognitive trajectories. Thirdly, we predicted that individuals with higher CR, as measured by intellectual ability and educational level, would experience 
less cognitive decline over time compared to individuals with low $C R$ in a sample of patients with $\mathrm{BP}$, similar to findings that suggest that $\mathrm{CR}$ is a mediator variable between brain injury/pathology and clinical outcomes. We also explored the effect of age on changes in cognitive test scores over time and expected that age would have a small but significant effect on changes on neuropsychological test scores over time; specifically, that older individuals would demonstrate a larger negative change in scores compared to younger participants. Additionally, we aimed to identify individuals who showed a significant decline in test scores ('decliners') over time and explored if any CR or clinical variables were associated with decline. Again, we hypothesized that low CR would be associated with higher rates of decline over the 5-year span.

\section{2 | METHODS}

\section{1 | Participants}

All participants were recruited from the Prechter Longitudinal Study of Bipolar Disorder, a large-scale naturalistic study of BP conducted at the University of Michigan in Ann Arbor, MI ${ }^{36,37}$ and approved by the University of Michigan institutional review board. For the present study, 159 individuals with BP (type I or II, or not otherwise specified [NOS]), and 54 healthy individuals without a history of psychiatric illness (HCs) were included based on having 5 years of longitudinal data available by July 2015. Two hundred and eighty-two participants were eligible for 5-year testing but did not complete it due to attrition or other factors (see Results section for analyses comparing those who completed 5-year testing and those who did not). Individuals were excluded from the longitudinal study if they had a history of diagnosed schizophrenia, active substance dependence, or a history of significant neurological disease at the time of study entry. $\mathrm{HC}$ participants were excluded from the study if they met diagnostic criteria for a DSM-IV-TR disorder at any time during the 5-year study timeframe, as assessed by biennial clinical interviews ( $n=11)$. Participant data were not used if evidence of substantial intellectual impairment (IQ $<70)$ was present $(n=1)$. Individuals with a history of developmental disorder were also screened out at the initial interview. Mood states during the neuropsychological assessment were also documented at the time at which they entered the study. At baseline, $54.1 \%$ of the BP group were euthymic, $36.5 \%$ were considered depressed, and $8.8 \%$ were considered to be in a hypo/manic state. All the HCs were in the euthymic state. Among the BP sample, separate chi-square analyses showed that mood state at the time of testing (i.e., depressed, manic, or euthymic) was not significantly related to overall decline $\left(\chi^{2}(2, N=158)=1.10, P=.578\right)$ or the number of tests on which the performance of participants declined $(F=.289, P=.75)$. Further, baseline test performances $(P>05)$ were not different among the specific mood states, other than the measure of visuospatial functioning (Rey-Osterrieth Complex Figure Test [RCFT] copy; F=5.39, $P=.005)$. Change in cognitive performance was not related to change in depression (Hamilton Depression Rating Scale ${ }^{38}$ [HDRS]) or mania symptoms (Young Mania Rating Scale ${ }^{39}$ [YMRS]) from baseline to year 5 (all $P>.05$ ). Further, results of ANOVA analyses showed that mood state at the time of testing was unrelated to change in cognitive performance across all tests. Thus, those with current mood symptoms were included to increase sample size.

\section{2 | Clinical assessment}

All participants completed an initial psychodiagnostic interview with an experienced clinician using the Diagnostic Interview for Genetic Studies $\left(\mathrm{DIGS}^{40}\right)$. Psychiatric diagnoses were then determined through best estimate consensus processes in which two $\mathrm{MD} / \mathrm{PhD}$ level clinicians independently evaluated information obtained from the DIGS and other available data (e.g., available medical records and self-report forms). Educational achievement and other demographic variables were obtained through the DIGS interview. Also, information regarding the presence of cerebrovascular disease/risk factors and historical clinical information (e.g., number of mood episodes, presence of psychosis and suicide history) were obtained through the DIGS. The HDRS $^{38}$ and YMRS $^{39}$ were used to assess mood state at the time of testing. The HDRS has 17 items and scores range from 0 to 50 , indicating overall depressive symptomatology. The YMRS has 11 items and scores range from 0 to 60 , indicating overall mania symptomatology. Each participant's medication classes and composite load score were determined with methods adapted from other groups, ${ }^{41-45}$ in which higher scores represent a larger medication burden.

\section{3 | Cognitive reserve variables}

Proxies for CR in our study included education and premorbid intellectual functioning as measured by the vocabulary subtest from the Wechsler Abbreviated Scale of Intelligence (see Neuropsychological Assessment below). Years of education was used as a continuous variable. Higher educational attainment and standardized vocabulary scores were considered to be reflective of higher CR.

\section{4 | Neuropsychological assessment}

All participants completed comprehensive neuropsychological testing at the time of their entry into the longitudinal study (baseline) and again 5 years later (year 5 ), administered by trained research associates and supervised by neuropsychologists on the team. The neuropsychological test battery was based on previous work by Langenecker et al. ${ }^{37}$ and captured neurocognitive abilities in the areas of attention, memory, language, processing speed, executive functioning, and motor speed. The test battery has also been used in a number of other published articles investigating neuropsychological abilities among individuals with $\mathrm{BP},{ }^{10,46-50}$ addressing separate questions about the disorder in cross-sectional analyses. Scores from seven neuropsychological tests were selected that emphasize major areas of cognitive dysfunction among individuals with BP. These included The California Verbal Learning Test-II (CVLT-II; long delay free recall: verbal memory), ${ }^{51}$ RCFT (copy, immediate, delay: visuospatial integration and visual memory), ${ }^{52}$ Purdue Pegboard (dominant and non-dominant hand speed: motor speed/dexterity), ${ }^{53}$ Wisconsin Card Sorting Test 
(WCST; total correct: executive functioning), ${ }^{54}$ Stroop Color and Word Test (Color-Word interference subtest: attention/executive functioning), ${ }^{55}$ Controlled Oral Word Association Test (COWAT; FAS administration: phonemic verbal fluency), ${ }^{56}$ Animal Fluency (Animals: semantic verbal fluency), ${ }^{56}$ and Trail Making Test A and B (TMTA and TMTB; psychomotor speed and executive functioning). The Wechsler Abbreviated Scale of Intelligence (WASI; overall intellectual ability) ${ }^{57}$ was administered during the baseline evaluation to provide information regarding overall intellectual ability (resulting in an estimated fullscale IQ score); however, we used the Vocabulary scaled score from the WASI as the measure of intelligence as this has been shown to be robust to neurological illness. ${ }^{58,59}$

\section{5 | Data analyses}

Analyses were completed using the IBM Statistical Package for Social Sciences (Version 21) and Statistical Analysis System. t-tests or chisquare tests were used to determine the significance of demographic differences between the BP and HC groups. Raw scores were converted to Z-scores based on mean $\mathrm{HC}$ group scores at baseline and year 5. Paired t-test analyses were used to compare the BP and HC groups in terms of their neuropsychological test scores at baseline, and again at year 5 . Change scores were created by calculating the difference in Z-scores between baseline testing and year 5 testing. Next, we identified decliners $(n=120)$ as individuals with change scores at least 1.5 standard deviations (SDs) below the $\mathrm{HC}$ mean on any of the cognitive tests. A change score of $<1.5$ SD below the $\mathrm{HC}$ mean was chosen as a cut-off to represent a clinical change in performance. Those who did not demonstrate a decline of $>1.5$ SD were considered non-decliners ( $n=104)$. T-test analyses of the decliners and non-decliners were completed to further assess the significance of differences among groups in terms of $C R$ variables. We used chi-square and t-test analyses to compare the BP decliners and non-decliners on clinical variables including cerebrovascular risk factor/disease variables. Finally, variable regression models were used to determine the relationship between changes in test scores over the 5 -year period, as well as the influence of $C R$ variables. As previously noted, $C R$ variables included in our analyses were education and IQ.

\section{3 | RESULTS}

There were no significant differences between the BP and $\mathrm{HC}$ groups in terms of education, age, gender, handedness, WASI full-scale IQ or WASI Vocabulary standard score at study entry. Descriptive statistics are presented in Table 1.

We compared participants who completed 5-year testing (completers; $n=283$ ) to those who did not complete it, despite being enrolled in the study for 5 years (non-completers; $n=282$ ) to contextualize this sample in terms of attrition. The completers had similar fullscale IQ $(P=.27)$ and education $(P=.19)$ to non-completers but were more likely to be older (mean(SD) $=38.97(13.13)$ vs mean(SD) $=35.90$ (14.11); $\mathrm{t}(237)=-2.68, P=.01)$ and male $(P=.0009)$.

Next, when differences between groups for neuropsychological test scores at baseline testing and year 5 testing were examined, the BP group showed significantly lower scores on seven of 13 test scores at baseline, and six of 13 test scores analyzed at year 5 as compared to the HC group. Please see Table 2.

To provide an indicator of overall relative change, change scores were created by calculating the difference in Z-scores for all neuropsychological tests between baseline testing and year 5 testing. See Figure 1. Change scores for the neuropsychological test variables did not differ between the BP and $\mathrm{HC}$ groups, with the exception of visuospatial skills (RCFT copy) and the delayed visual memory score (RCFT delay), which revealed an improvement in performance for BP patients, but not HCs $(t=-2.07, P=.04$ and $t=-2.70, P=.007$, respectively; see Table 3). Immediate visual memory (RCFT immediate) showed a trend toward significance $(t=-1.97, P=.05)$.

Individual participants were considered 'decliners' if they obtained a change score that was at least 1.5 SDs below the mean $\mathrm{HC}$ change
TABLE 1 Demographic variables; mean and standard deviation (SD)

\begin{tabular}{|lllll|}
\hline & $\begin{array}{l}\text { Bipolar disorder I, II, } \\
\text { NOS: mean (SD)/\% }\end{array}$ & $\begin{array}{l}\text { Healthy controls: } \\
\text { mean (SD)/\% }\end{array}$ & $\begin{array}{l}\text { Value (t or } \\
\text { chi-square) }\end{array}$ & $P$ \\
\hline Demographics & & & & \\
\hline Education & $15.33(2.32)$ & $15.85(2.17)$ & 1.44 & .15 \\
\hline Gender (female) & $74 \%$ & $63 \%$ & 2.20 & .14 \\
\hline Age & $40.70(12.03)$ & $36.78(14.88)$ & -1.95 & .05 \\
\hline Right hand dominance & $86.1 \%$ & $86.8 \%$ & 0.02 & .90 \\
\hline WASI Vocabulary SS & $12.65(2.74)$ & $12.40(2.99)$ & -0.55 & .59 \\
\hline Clinical variables & & & & \\
\hline HDRS (baseline) & $8.92(6.18)$ & $1.14(1.53)^{*}$ & -8.96 & $<0.001^{*}$ \\
\hline HDRS (year 5) & $8.26(5.54)$ & $1.85(3.29)^{*}$ & -8.02 & $<0.001^{*}$ \\
\hline YMRS (baseline) & $2.70(3.43)$ & $0.34(0.71)^{*}$ & -4.94 & $<0.001^{*}$ \\
\hline YMRS (year 5) & $3.27(4.06)$ & $0.93(2.67)^{*}$ & -3.96 & $<0.001^{*}$ \\
\hline
\end{tabular}

*Significantly different at $P<.05$. NOS, bipolar disorder not otherwise specified; WASI, Wechsler Abbreviated Scale of Intelligence; SS, scaled score; HRDS, Hamilton Depression Rating Scale; YMRS, Young Mania Rating Scale. 
TABLE 2 Independent t-test results comparing neuropsychological test scores for bipolar disorder (BP) and healthy control $(\mathrm{HC})$ groups at baseline and year 5 visits

\begin{tabular}{|c|c|c|c|c|}
\hline Measure & $\begin{array}{l}\text { BP I, II, NOS: } \\
\text { mean (SD); raw } \\
\text { score }\end{array}$ & $\begin{array}{l}\text { Healthy } \\
\text { controls: } \\
\text { mean (SD); } \\
\text { raw score }\end{array}$ & $\mathrm{t}$ value & $\operatorname{Pr}>|t|$ \\
\hline \multicolumn{5}{|c|}{ Visuospatial integration and visual memory } \\
\hline \multicolumn{5}{|c|}{ RCFT copy } \\
\hline Baseline & $32.07(3.31)$ & $33.19(2.52)$ & 2.29 & $.023^{*}$ \\
\hline 5 year & 33.55 (3.41) & $33.75(2.66)$ & 0.39 & .70 \\
\hline \multicolumn{5}{|c|}{ RCFT immediate } \\
\hline Baseline & $18.82(7.06)$ & $22.72(5.87)$ & 3.66 & $.000^{*}$ \\
\hline 5 year & 21.65 (7.37) & $24.84(7.53)$ & 2.72 & $.007^{*}$ \\
\hline \multicolumn{5}{|l|}{ RCFT delay } \\
\hline Baseline & $18.43(6.82)$ & $22.53(5.87)$ & 3.95 & $.000^{*}$ \\
\hline 5 year & $21.56(7.51)$ & $23.82(6.89)$ & 1.95 & .052 \\
\hline \multicolumn{5}{|c|}{ Verbal memory } \\
\hline \multicolumn{5}{|c|}{ CVLT total learning } \\
\hline Baseline & $52.94(10.64)$ & $54.53(11.03)$ & 0.93 & .35 \\
\hline 5 year & $51.39(11.51)$ & $52.54(13.62)$ & 0.60 & .55 \\
\hline \multicolumn{5}{|c|}{ CLVT short delay } \\
\hline Baseline & $11.25(3.18)$ & $11.79(3.15)$ & 1.09 & .28 \\
\hline 5 year & $11.03(3.66)$ & $11.43(3.91)$ & 0.67 & .50 \\
\hline \multicolumn{5}{|c|}{ CVLT long delay } \\
\hline Baseline & 11.67 (2.99) & $12.23(2.97)$ & 1.18 & .24 \\
\hline 5 year & $11.56(3.60)$ & $11.59(4.11)$ & 0.05 & .96 \\
\hline \multicolumn{5}{|c|}{ Motor dexterity } \\
\hline \multicolumn{5}{|c|}{ Peg dominant } \\
\hline Baseline & $14.43(1.87)$ & $15.62(2.20)$ & 3.86 & $.000^{*}$ \\
\hline 5 year & $14.57(2.30)$ & $15.96(2.37)$ & 3.78 & $.000^{*}$ \\
\hline \multicolumn{5}{|c|}{ Peg non-dominant } \\
\hline Baseline & $13.36(1.88)$ & $14.65(1.58)$ & 4.54 & $.000^{*}$ \\
\hline 5 year & $13.68(2.23)$ & $15.02(2.17)$ & 3.83 & $.000^{*}$ \\
\hline \multicolumn{5}{|c|}{ WCST correct } \\
\hline Baseline & 70.17 (13.46) & 71.29 (11.38) & 0.49 & .62 \\
\hline 5 year & $69.27(10.89)$ & $68.87(10.26)$ & -0.24 & .81 \\
\hline \multicolumn{5}{|l|}{ Stroop } \\
\hline Baseline & $0.74(7.03)$ & $4.16(8.86)$ & 2.88 & $.004^{*}$ \\
\hline 5 year & $2.79(7.09)$ & $6.56(6.88)$ & 3.39 & $.001^{*}$ \\
\hline \multicolumn{5}{|l|}{ COWAT } \\
\hline Baseline & 41.84 (13.07) & $42.51(10.56)$ & 0.34 & .74 \\
\hline 5 year & 39.06 (13.28) & $42.22(12.75)$ & 1.53 & .13 \\
\hline \multicolumn{5}{|l|}{ Animals } \\
\hline Baseline & $20.52(5.90)$ & 41.84 (13.07) & 0.53 & .60 \\
\hline 5 year & $18.13(5.82)$ & $19.67(4.97)$ & 1.74 & .08 \\
\hline \multicolumn{5}{|l|}{ TMTA } \\
\hline Baseline & 30.70 (11.75) & $27.26(12.68)$ & -1.81 & .07 \\
\hline 5 year & $29.16(11.15)$ & $24.06(10.51)$ & -2.95 & $.004^{*}$ \\
\hline
\end{tabular}

TABLE 2 (Continued)

\begin{tabular}{clllll} 
& $\begin{array}{l}\text { BP I, II, NOS: } \\
\text { mean (SD); raw } \\
\text { score }\end{array}$ & $\begin{array}{l}\text { Healthy } \\
\text { controls: } \\
\text { mean (SD); } \\
\text { raw score }\end{array}$ & t value & $\operatorname{Pr}>|t|$ \\
\hline TMTB & & & & \\
\hline Baseline & $78.48(44.00)$ & $59.64(27.74)$ & -2.90 & $.004^{*}$ \\
\hline 5 year & $80.26(40.68)$ & $60.11(27.07)$ & 3.36 & $.001^{*}$ \\
\hline
\end{tabular}

*Significant at $P \leq .05$. RCFT copy, Rey-Osterrieth Complex Figure Test copy; RCFT immediate, Rey-Osterrieth Complex Figure Test immediate memory; RCFT delay, Rey-Osterrieth Complex Figure Test delay memory; Peg dominant, Perdue Pegboard speed dominant hand; Peg non-dominant, Perdue Pegboard speed non-dominant hand; CVLT total learning, California Verbal Learning Test total learning; CVLT short delay, California Verbal Learning Test short delay free recall; CVLT long delay, California Verbal Learning Test long delay free recall; WCST, Wisconsin Card Sort Test, total correct; Stroop, Stroop Color/Word interference total; COWA, Controlled Oral Word Association Test; Animals, Semantic Fluency, Animals; TMTA, Trail Making Test A; TMTB, Trail Making Test B; NOS, bipolar disorder not otherwise specified.

score on one or more neuropsychological tests (i.e., if they demonstrated more change in test scores over 5 years than was typical for the HC sample). Individual HC means and SDs were used to calculate change scores for each test score, at each time point. 'Non-decliners' included individuals with change scores within the $\mathrm{HC}$ range, based on the $\mathrm{HC}$ change score mean (i.e., those who did not change more drastically than the average $\mathrm{HC}$ over the 5-year timeframe).

Decliners included 74 individuals from the BP group (46.5\% of the $\mathrm{BP}$ group) and 20 individuals from the $\mathrm{HC}$ group (37\% of the group). Within the decliners, there were no significant differences between the BP and $\mathrm{HC}$ groups in terms of age, education, WASI Vocabulary (estimated verbal IQ) or gender. Within the BP group only, decliner status was not associated with any of the demographic or CR variables. However, we found that the decliners in the BP group had significantly lower overall IQ scores than those in the BP non-decliner group $(P<.001)$.

Those in the decliner group included a majority who declined on at least one cognitive test $(n=53,71.6 \%), 20.3 \%$ who declined on two tests ( $n=15$ ), $4.1 \%$ who declined on three tests $(n=3), 2.7 \%$ who declined on four tests $(n=2)$ and $1.4 \%$ who declined on six tests $(n=1)$. Within the $\mathrm{HC}$ group only, decliner status was associated with education, with the decliner group having less education than the nondecliners ( $P=.005$, decliners education=M 14.8 [SD 2.4]; non-decliners education=M 16.5 [SD 1.8]). Of the HCs who declined, the majority declined on one cognitive test ( $n=13 ; 65 \%), 20 \%$ declined on two tests $(n=4)$ and $15 \%$ declined on three tests $(n=3)$.

Although not the main analyses, we examined differences in clinical illness variables between decliners and non-decliners among the individuals with BP. See Table S1. There were no illness features that were significantly different between the decliner and non-decliner groups. Further, there were no differences between the decliners and non-decliners in terms of the presence of cerebrovascular disease or cerebrovascular risk factors. There also was no difference in total medication load when comparing BP decliners to BP non-decliners. When 


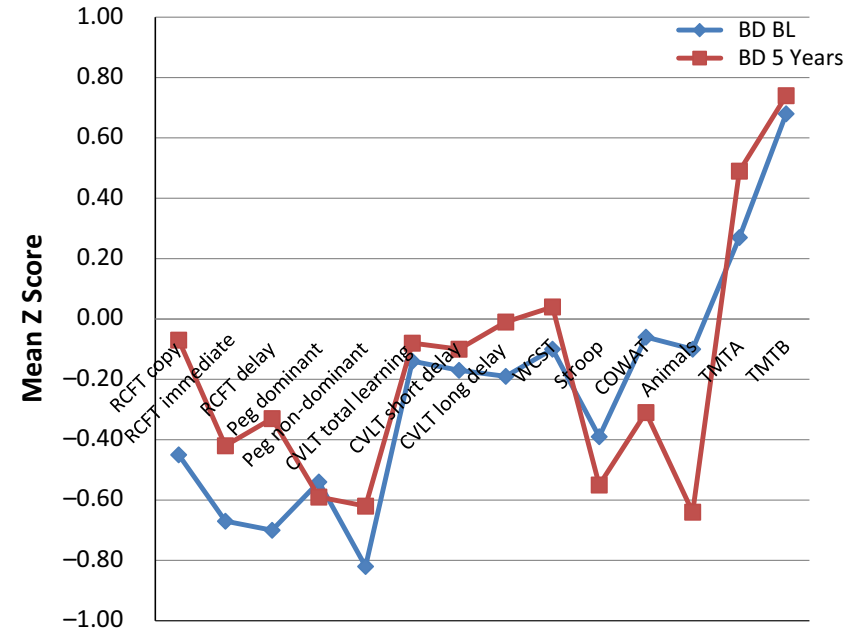

FIGURE 1 Neuropsychological test score means at baseline $(\mathrm{BL})$ and 5 years for patients with bipolar disorder (BP) (Z-scores). All healthy control $(\mathrm{HC})$ data are set to a Z-score of 0. RCFT copy, Rey-Osterrieth Complex Figure Test copy; RCFT immediate, ReyOsterrieth Complex Figure Test immediate memory; RCFT delay, Rey-Osterrieth Complex Figure Test delay memory; Peg dominant, Perdue Pegboard speed dominant hand; Peg non-dominant, Perdue Pegboard speed non-dominant hand; CVLT total learning, California Verbal Learning Test total learning; CVLT short delay, California Verbal Learning Test short delay free recall; CVLT long delay, California Verbal Learning Test long delay free recall; WCST, Wisconsin Card Sort Test, total correct; Stroop, Stroop Color/Word interference total; COWA, Controlled Oral Word Association Test; Animals, Semantic Fluency, Animals; TMTA, Trail Making Test A; TMTB, Trail Making Test $\mathrm{B}$ [Colour figure can be viewed at wileyonlinelibrary.com]

considering the entire sample (HCs and BP patients), the results continued to be non-significant.

Finally, the changes in neuropsychological performance over 5 years were analyzed at a post hoc level with linear regression modeling. The BP and HC groups were analyzed separately to evaluate the influences of IQ and education. Regression models showed that, within the $\mathrm{HC}$ group, age and baseline verbal IQ influenced change in semantic verbal fluency performance (Animals). Specifically, education influenced semantic fluency (parameter estimate $=0.11, P=.02$ ) and with every 10-point increase in IQ, change in semantic verbal fluency scores decreased by 0.08 (parameter estimate $=-0.08, P=.02$ ). Further, baseline verbal IQ influenced change in interference control (Stroop parameter estimate=-0.94, $P=.02$ ) such that, for every 10-point increase in IQ, change in interference decreased by 0.94. Age and education influenced visuomotor attention (TMTA; parameter estimate age $=0.03, P=.004$; parameter estimate education $=-0.13, P=.03$ ) and fine motor dexterity (parameter estimate age $=-0.03, P=.008$; parameter estimate education $=0.17, P=.02$ ). Within the BP group, regression analyses indicated that lower IQ score was associated with significantly decreased verbal memory scores (CVLT long delay free recall; parameter estimate $=-0.06, P=.01)$. Lower education was found to be related to attenuated decline on a visuospatial functioning test (RCFT copy; parameter estimate $=-0.10, P=.04$ ) and simple visuomotor attention test (TMTA parameter estimate $=-.07, P=.048$ ). Age at the time of testing was also included in regression analyses as a covariate, and results indicated that age was related to change on specific measures (see Table 4).

\section{4 | DISCUSSION}

The results of this study yielded several notable findings regarding change in cognition over 5 years in individuals diagnosed with BP, compared to control subjects, as well as important insight into potential CR factors, particularly in those with $\mathrm{BP}$, using a large sample size and longitudinal study design. First, we re-demonstrated that baseline neurocognitive deficits exist among those with BP compared to HCs, consistent with previous literature. ${ }^{37}$ Specifically, we showed that significantly lower scores were obtained by individuals with BP in terms of visuospatial ability, immediate and delayed visual memory, bilateral motor speed, attention, executive functioning, and psychomotor speed (at baseline, year 5, or both time points). Next, we found that change in neurocognitive performance over time did not differ between the BP and $\mathrm{HC}$ groups (with one exception, which showed slightly improved performance for BP patients in delayed visual memory). More broadly, we did not find evidence for accelerated decline in the 5-year timeframe for BP patients, consistent with an interpretation of additive, but not compounded or accelerated, effects of age and BP. ${ }^{60}$ When we identified individuals who demonstrated a significant decline on at least one cognitive measure, we found that decline was significantly associated with lower overall intellectual scores compared to those who did not decline within the BP group only. This finding was not replicated within the $\mathrm{HC}$ decliner group. However, we did not find a difference between the BP decliners and BP non-decliners in terms of our CR variables (WASI Vocabulary and education). This finding suggests that overall IQ may be protective for general cognitive decline in $\mathrm{BP}$, but not traditional $\mathrm{CR}$ variables as expected.

Instead, we found verbal IQ (WASI Vocabulary) and education were related to changes in specific cognitive domains. Specifically, we found that intellectual ability was related to change in performance in terms of visuospatial ability, simple visuomotor attention, and verbal memory in the BP group, three particularly important skills in everyday life. Overall, findings supported our hypotheses and indicate that there does not seem to be evidence of accelerated cognitive decline in $\mathrm{BP}$, and that $\mathrm{CR}$ variables were related to change in select areas of cognition. The overall lack of cognitive decline could represent a stable cognitive endophenotype in BP and warrants future exploration. ${ }^{46,61}$

Overall, our sample was highly educated and intelligent, with IQ scores well into the average and high-average ranges, which may account for why we did not find change in more of the cognitive domains assessed. Additionally, a longer period of time, beyond 5 years, may be necessary to detect more significant cognitive change within the groups. Since our cohort was relatively young (mean age $=41.6$ years), longer studies and analysis of older individuals with BP are warranted. It is possible that older individuals with BP do experience accelerated cognitive decline and that our sample was too young to allow us to detect this. 


\begin{tabular}{|c|c|c|c|}
\hline Measure & BP I, II, NOS: mean (SD) & Healthy controls: mean (SD) & $\operatorname{Pr}>|t|$ \\
\hline \multicolumn{4}{|c|}{ Visuospatial integration and visual memory } \\
\hline RCFT copy & $0.37(1.24)$ & $0.00(0.79)$ & $0.39^{*}$ \\
\hline RCFT immediate & $0.24(0.96)$ & $-0.04(0.71)$ & 0.05 \\
\hline RCFT delay & $0.37(0.92)$ & $0.00(0.70)$ & $0.007^{*}$ \\
\hline \multicolumn{4}{|l|}{ Verbal memory } \\
\hline CVLT total learning & $0.06(0.72)$ & $0.00(0.88)$ & 0.64 \\
\hline CVLT short delay & $0.08(0.85)$ & $0.00(0.81)$ & 0.52 \\
\hline CVLT long delay & $0.20(0.78)$ & $-0.01(0.81)$ & 0.11 \\
\hline \multicolumn{4}{|l|}{ Motor speed/dexterity } \\
\hline Peg dominant & $-0.04(0.76)$ & $-0.01(1.13)$ & 0.80 \\
\hline Peg non-dominant & $0.21(0.93)$ & $0.00(0.98)$ & 0.16 \\
\hline \multicolumn{4}{|l|}{ Executive functioning } \\
\hline WCST correct & $0.07(1.75)$ & $-0.08(1.51)$ & 0.61 \\
\hline Stroop & $-0.16(1.00)$ & $0.00(0.85)$ & 0.31 \\
\hline \multicolumn{4}{|l|}{ Verbal fluency } \\
\hline COWAT & $-0.57(2.08)$ & $-0.10(1.90)$ & 0.14 \\
\hline Animals & $-0.04(0.71)$ & $-0.22(1.09)$ & 0.28 \\
\hline \multicolumn{4}{|c|}{ Psychomotor speed/executive functioning } \\
\hline TMTA & $0.22(0.87)$ & $0.01(1.07)$ & 0.17 \\
\hline TMTB & $0.08(1.28)$ & $0.09(0.74)$ & 0.94 \\
\hline
\end{tabular}

TABLE 3 Independent t-test comparing change scores among bipolar disorder (BP) and healthy control $(\mathrm{HC})$ groups

*Significant at $P \leq .05$. NOS, BP not otherwise specified; SD, standard deviation; RCFT copy, ReyOsterrieth Complex Figure Test copy; RCFT immediate, Rey-Osterrieth Complex Figure Test immediate memory; RCFT delay, Rey-Osterrieth Complex Figure Test delay memory; Peg dominant, Perdue Pegboard speed dominant hand; Peg non-dominant, Perdue Pegboard speed non-dominant hand; CVLT total learning, California Verbal Learning Test total learning; CVLT short delay, California Verbal Learning Test short delay free recall; CVLT long delay, California Verbal Learning Test long delay free recall; WCST, Wisconsin Card Sort Test, total correct; Stroop, Stroop Color/Word interference total; COWA, Controlled Oral Word Association Test; Animals, Semantic Fluency, Animals; TMTA, Trail Making Test A; TMTB, Trail Making Test B.

Our finding that those with a bipolar diagnosis did not experience an increased decline over 5 years is in support of a growing body of new literature, ${ }^{11-13}$ and also contradictory to previous work that has claimed that BP is associated with significant neurocognitive decline. ${ }^{5-9,62}$ However, our study utilized a large sample of participants with BP and longitudinal methods, which may increase the specificity and generalizability of our findings to BP groups, given the typical high rate of variability between patients. Overall, this finding is important to consider in treatment planning and support for middle-aged and older adults with BP, as these individuals may start at a lower cognitive level, and, with a similar pattern of decline, may reach the point of dementia at an earlier age. Specifically, the BP group tended to have lower baseline neuropsychological scores compared to HCs, and so a 'normal' decline in cognition associated with age may lead to more significant functional deficits as they may reach an objectively more impaired cognitive level, which might also translate into higher levels of occupational disability. ${ }^{14}$

Our study makes a significant contribution to the existing literature in that it applies the concept of CR to BP, a highly unique condition with a complex constellation of neuropsychological symptoms and mood difficulties. Our findings suggest, to some degree, that higher intellectual abilities earlier in life may be related to preserved cognitive abilities in BP, while traditional CR variables, including verbal IQ and education, have a selective effect on visual spatial ability and verbal memory. It is possible that CR may provide the same kind of preservation and/or accommodation of neurocognitive difficulties in BP as that demonstrated in stroke, traumatic brain injury (TBI), dementia, or other conditions affecting cognition. To our knowledge, this is the largest study utilizing a longitudinal design following patients for 5 years that exists in the current literature. Although other studies have explored the relationship between $\mathrm{CR}$ variables and neuropsychological performance in $\mathrm{BP}$, or change in neuropsychological test scores for patients with BP over time, this is the first study to our knowledge to integrate both features.

While the current study has a number of strengths, there are a few limitations that may reduce the generalizability of our results and should be considered for future studies. In particular, this study utilized participants taking a variety of psychotropic medications and participating in psychosocial treatments for BP and with comorbid conditions, as is common in naturalistic studies of this kind. It is possible that specific medications, such as lithium, had an effect on neurocognitive functioning in our study, but since we did not detect significant 
TABLE 4 Regression results showing the significant effect of age on neuropsychological test scores in our sample (all participants).

\begin{tabular}{|c|c|c|c|c|c|c|}
\hline Measure & Group & df & Parameter estimate & Standard error & $\mathrm{t}$ value & $\operatorname{Pr}>|t|$ \\
\hline RCFT copy & $\mathrm{BP}$ & 1 & 0.015 & 0.008 & 1.77 & 0.08 \\
\hline \multirow[t]{2}{*}{ RCFT immediate } & $\mathrm{BP}$ & 1 & 0.009 & 0.006 & 1.39 & 0.17 \\
\hline & $\mathrm{HC}$ & 1 & 0.001 & 0.007 & 0.10 & 0.93 \\
\hline RCFT delay & $\mathrm{HC}$ & 1 & 0.003 & 0.007 & 0.44 & 0.66 \\
\hline \multicolumn{7}{|l|}{ Verbal memory } \\
\hline \multirow[t]{2}{*}{ CVLT total learning } & $\mathrm{BP}$ & 1 & 0.003 & 0.005 & 0.67 & 0.50 \\
\hline & $\mathrm{HC}$ & 1 & 0.004 & 0.008 & 0.45 & 0.66 \\
\hline CVLT long delay & $\mathrm{HC}$ & 1 & 0.010 & 0.008 & 1.29 & 0.20 \\
\hline \multicolumn{7}{|l|}{ Motor speed/dexterity } \\
\hline \multirow[t]{2}{*}{ Peg dominant } & $\mathrm{BP}$ & 1 & -0.016 & 0.005 & -3.12 & $0.002^{*}$ \\
\hline & $\mathrm{HC}$ & 1 & -0.027 & 0.010 & -2.78 & $0.008^{*}$ \\
\hline \multirow[t]{2}{*}{ Peg non-dominant } & $\mathrm{BP}$ & 1 & -0.012 & 0.006 & -1.90 & 0.06 \\
\hline & $\mathrm{HC}$ & 1 & -0.019 & 0.009 & -2.18 & $0.03^{*}$ \\
\hline \multicolumn{7}{|l|}{ Executive functioning } \\
\hline \multirow[t]{2}{*}{ WCST correct } & $\mathrm{BP}$ & 1 & 0.009 & 0.012 & 0.75 & 0.46 \\
\hline & $\mathrm{HC}$ & 1 & 0.018 & 0.015 & 1.17 & 0.25 \\
\hline Animals & $\mathrm{HC}$ & 1 & 0.003 & 0.007 & 0.52 & 0.61 \\
\hline \multicolumn{7}{|c|}{ Psychomotor speed/executive functioning } \\
\hline \multirow[t]{2}{*}{ TMTA } & $\mathrm{BP}$ & 1 & 0.015 & 0.006 & 2.51 & $0.013^{*}$ \\
\hline & $\mathrm{HC}$ & 1 & 0.025 & 0.008 & 3.05 & $0.004^{*}$ \\
\hline \multirow[t]{2}{*}{ TMTB } & $\mathrm{BP}$ & 1 & 0.011 & 0.009 & 1.21 & 0.23 \\
\hline & $\mathrm{HC}$ & 1 & 0.000 & 0.007 & -0.02 & 0.98 \\
\hline
\end{tabular}

*Significant at $P \leq .05$. BP, bipolar disorder; HC, healthy control; RCFT copy, Rey-Osterrieth Complex Figure Test copy; RCFT immediate, Rey-Osterrieth Complex Figure Test immediate memory; RCFT delay, Rey-Osterrieth Complex Figure Test delay memory; Peg dominant, Perdue Pegboard speed dominant hand; Peg non-dominant, Perdue Pegboard speed non-dominant hand; CVLT total learning, California Verbal Learning Test total learning; CVLT short delay, California Verbal Learning Test short delay free recall; CVLT long delay, California Verbal Learning Test long delay free recall; WCST, Wisconsin Card Sort Test, total correct; Stroop, Stroop Color/Word interference total; COWA, Controlled Oral Word Association Test; Animals, Semantic Fluency, Animals; TMTA, Trail Making Test A; TMTB, Trail Making Test B.

differences between changes in BP and HC groups over time, it is unlikely that medications were related to a significant decline in cognition. Please see Table S1, which illustrates the total medication load BP participants reported at the time of their initial assessment. Also, the particular neuropsychological test battery utilized in our study was selected for ease of administration and comprehensiveness across cognitive domains known to be affected in $\mathrm{BP} .{ }^{63}$ Administration time and participant fatigue generally precluded a longer or more comprehensive test battery. Finally, we included BP participants in various mood states in our analyses (euthymic, depressed, and manic), and acute mood symptoms may have influenced overall performance on testing. However, our preliminary analyses did not find a relationship between decline and mood state, and therefore it seems unlikely that mood at the time of testing significantly influenced our findings. Future work 
may focus on further characterizing the BP participants who showed evidence of decline over our 5-year timeframe. Use of additional statistical methods (i.e., cluster analyses, etc.) may provide evidence of decliner subtypes, offering more precise ways to identify those in need of future support. Other factors such as substance abuse, history of traumatic brain injury, illness duration, or cardiovascular risk are also important considerations for future research, although these were beyond the scope of our current work.

It is possible that our findings were influenced by test-retest variability in our sample. However, our decision to use a change score cutoff of 1.5 SD below the mean makes it likely that our findings reflect meaningful differences, particularly as we utilized standard neuropsychological measures that are widely accepted in the field and report strong test-retest reliability.

In summary, the present study demonstrated that neurocognitive deficits exist among individuals with BP, though there is generally a high degree of heterogeneity and variability among patients. These deficits seem to be present early in the disorder, but do not progress at a more rapid rate than that experienced by a 'normally' aging healthy adult sample with no psychiatric illness. Further, higher intellectual ability may selectively preserve cognitive abilities over time, particularly for individuals with BP and an above-average IQ. Traditional CR factors (WASI Vocabulary and education) were only partially influential for decline. We would like to emphasize that it is quite difficult to examine $C R$ variables in this population, as the accumulation of $C R$ may be directly impacted by the presentation of disruptive psychological symptoms at a young age.

Our findings provide valuable insight for providers and family members working with patients experiencing BP cognitive symptoms. Specifically, cognitive training groups or other rehabilitation treatments may be quite beneficial for some BP subgroups, and perhaps less necessary for others. Overall, our findings highlight the importance of intellectual engagement in those with neuropsychiatric illness, which may provide some defense against functional decline with age. This is only a speculation based on a small, but growing, body of literature, however, and significantly more research is needed in this area for continued understanding of the relationship between premorbid intellectual ability, neurocognitive symptoms of BP, and functional decline over time.

\section{ACKNOWLEDGEMENTS}

This research was supported by the Heinz C. Prechter Bipolar Research Fund at the University of Michigan Depression Center and the Richard Tam Foundation. We would like to express appreciation to our research participants in the Prechter Longitudinal Study of Bipolar Disorder. We would also like to acknowledge and thank the Prechter Bipolar Research Team for their contributions to this project.

\section{DISCLOSURES}

Kristin Hinrichs, Rebecca Easter, Kaley Angers, Bethany Pester, Zongshan Lai, David Marshall, Melvin Mclnnis, Scott Langenecker, and
Kelly Ryan do not report any disclosures. Masoud Kamali has received grant funding from Janssen Pharmaceutical and Assurex Health.

\section{REFERENCES}

1. Lewandowski KE, Cohen BM, Ongur D. Evolution of neuropsychological dysfunction during the course of schizophrenia and bipolar disorder. Psychol Med. 2011;41:225-241.

2. Kurtz MM, Gerraty RT. A meta-analytic investigation of neurocognitive deficits in bipolar illness: profile and effects of clinical state. Neuropsychology. 2009;23:551-562.

3. Rubinsztein JS, Michael A, Paykel ES, Sahakian BJ. Cognitive impairment in remission in bipolar affective disorder. Psychol Med. 2000;30:1025-1036.

4. Robinson LJ, Thompson JM, Gallagher P, et al. A meta-analysis of cognitive deficits in euthymic patients with bipolar disorder. J Affect Disord. 2006;93:105-115.

5. Kessing LV, Andersen PK. Does the risk of developing dementia increase with the number of episodes in patients with depressive disorder and in patients with bipolar disorder? J Neurol Neurosurg Psychiatry. 2004;75:1662-1666.

6. Kessing LV, Nilsson FM. Increased risk of developing dementia in patients with major affective disorders compared to patients with other medical illnesses. J Affect Disord. 2003;73:261-269.

7. Kessing LV, Olsen EW, Mortensen PB, Andersen PK. Dementia in affective disorder: a case-register study. Acta Psychiatr Scand. 1999;100:176-185.

8. Berk M. Neuroprogression: pathways to progressive brain changes in bipolar disorder. Int J Neuropsychopharmacol. 2009;12:441-445.

9. Martino DJ, Strejilevich SA, Scápola M, et al. Heterogeneity in cognitive functioning among patients with bipolar disorder. J Affect Disord. 2008;109:149-156.

10. Weisenbach SL, Marshall D, Weldon AL, et al. The double burden of age and disease on cognition and quality of life in bipolar disorder. Int J Geriatr Psychiatry. 2014;29:952-961.

11. Gildengers A, Chisholm D, Butters M, et al. Two-year course of cognitive function and instrumental activities of daily living in older adults with bipolar disorder: evidence for neuroprogression? Psychol Med. 2013;43:801-811.

12. Santos JL, Aparicio A, Bagney A, et al. A five-year follow-up study of neurocognitive functioning in bipolar disorder. Bipolar Disord. 2014;16:722-731.

13. Strejilevich SA, Samame C, Martino DJ. The trajectory of neuropsychological dysfunctions in bipolar disorders: a critical examination of a hypothesis. J Affect Disord. 2015;175:396-402.

14. Ryan KA, Vederman AC, Kamali M., et al. Emotion perception and executive functioning predict work status in euthymic bipolar disorder. Psychiatry Res 2013;210:472-478.

15. Ryan KA, Vederman AC, McFadden EM, et al. Executive functioning components change across phases of bipolar disorder. Bipolar Disord. 2012;14:527-536.

16. Richards M, Deary IJ. A life course approach to cognitive reserve: a model for cognitive aging and development? Ann Neurol. 2005;58:617-622.

17. Tucker AM, Stern Y. Cognitive reserve in aging. Curr Alzheimer Res. 2011;8:354-360.

18. Valenzuela MJ, Sachdev P. Brain reserve and cognitive decline: a nonparametric systematic review. Psychol Med. 2006;36:1065-1073.

19. Mortimer JA. Brain reserve and the clinical expression of Alzheimer's disease. Geriatrics. 1997;52:1p.

20. Stern Y. Cognitive reserve. Neuropsychologia. 2009;47:2015-2028.

21. Bieliauskas LA, Back-Madruga C, Lindsay KL, et al. Clinical relevance of cognitive scores in hepatitis $C$ patients with advanced fibrosis. $J$ Clin Exp Neuropsychol. 2006;28:1346-1361. 
22. Katzman R. Education and the prevalence of dementia and Alzheimer's disease. Neurology. 1993;43:13-20.

23. Satz $\mathrm{P}$, Morgenstern $\mathrm{H}$, Miller EN, et al. Low education as a possible risk factor for cognitive abnormalities in HIV-1: findings from the multicenter AIDS Cohort Study (MACS). J Acquir Immune Defic Syndr. 1993;6:503-511.

24. Stern Y, Gurland B, Tatemichi TK, Tang MX, Wilder D, Mayeux R. Influence of education and occupation on the incidence of Alzheimer's disease. J Am Med Assoc. 1994;271:1004-1010.

25. Meng X, D'Arcy C. Education and dementia in the context of the cognitive reserve hypothesis: a systematic review with meta-analyses and qualitative analyses. PLoS ONE. 2012;7:e38268.

26. Mortimer JA, Snowdon DA, Markesbery WR. Head circumference, education and risk of dementia: findings from the nun study. J Clin Exp Neuropsychol. 2003;25:671-679.

27. Albert MS, Jones K, Savage CR, et al. Predictors of cognitive change in older persons: MacArthur studies of successful aging. Psychol Aging. 1995; 10:578-589.

28. Hinrichs KH, Hayek A, Kalmback DA, Gabel NM, Bieliauskas LA. Cognitive reserve and executive function: impact on judgment of health and safety. J Rehabil Res Dev. 2016;53:863-872.

29. Barnett JH, Salmond CH, Jones PB, Sahakian BJ. Cognitive reserve in neuropsychiatry. Psychol Med. 2006;36:1053-1064.

30. Scott JC, Matt GE, Wrocklage KM, et al. A quantitative meta-analysis of neurocognitive functioning in posttraumatic stress disorder. Psychol Bull. 2015;141:105-140.

31. Forcada I, Mur M, Mora E, Vieta E, Bartres-Faz D, Portella MJ. The influence of cognitive reserve on psychosocial and neuropsychological functioning in bipolar disorder. Eur Neuropsychopharmaco. 2014;25:214-222.

32. Anaya C, Torrent C, Caballero FF, Vieta E, Bonnin Cdel M, AyusoMateos JL. Cognitive reserve in bipolar disorder: relation to cognition, psychosocial functioning and quality of life. Acta Psychiatr Scand. 2016;133:386-398.

33. Sanchez-Moreno J, Martinez-Aran A, Tabares-Seisdedos R, Torrent C, Vieta E, Ayuso-Mateos JL. Functioning and disability in bipolar disorder: an extensive review. Psychother Psychosom. 2009;78:285-297.

34. Kumar CS, Frangou S. Clinical implications of cognitive function in bipolar disorder. Ther Adv Chronic Dis. 2010;1:85-93.

35. Burgess B, Curtis-Downes D, Gibson RC. Education and employment levels among Jamaican patients newly diagnosed with schizophrenia and bipolar disorder. Int J Soc Psychiatry. 2012;59:247-253.

36. Langenecker SA, Caveney AF, Giordani B, et al. The sensitivity and psychometric properties of a brief computer-based cognitive screening battery in a depression clinic. Psychiatry Res. 2007;152:143-154.

37. Langenecker SA, Saunders EFH, Kade AM, Ransom MT, McInnis MG. Intermediate cognitive phenotypes in bipolar disorder. J Affect Disord. 2010;122:285-293.

38. Hamilton MAX. Development of a rating scale for primary depressive illness. Br J Soc Clin Psychol. 1967;6:278-296.

39. Young RC, Biggs JT, Ziegler VE, Meyer DA. A rating scale for mania: reliability, validity and sensitivity. BrJ Psychiatry. 1978;133:429-435.

40. Nurnberger JI Jr, Blehar MC, Kaufmann CA, et al. Diagnostic interview for genetic studies. Rationale, unique features, and training. NIMH Genetics Initiative. Arch Gen Psychiatry. 1994;51:849-859; discussion 63-4.

41. Almeida JRC, Akkal D, Hassel S, et al. Reduced gray matter volume in ventral prefrontal cortex but not amygdala in bipolar disorder: significant effects of gender and trait anxiety. Psychiatry Res. 2009;171:54-68.

42. Davis JM, Chen N. Dose response and dose equivalence of antipsychotics. J Clin Psychopharmacol. 2004;24:192-208.

43. Hassel S, Almeida JRC, Kerr N, et al. Elevated striatal and decreased dorsolateral prefrontal cortical activity in response to emotional stimuli in euthymic bipolar disorder: no associations with psychotropic medication load. Bipolar Disord. 2008;10:916-927.

44. Sackeim HA. The definition and meaning of treatment-resistant depression. J Clin Psychiatry. 2001;62:10-17.

45. Sackeim HA. The definition and meaning of treatment-resistant depression. J Clin Psychiatry. 2001;62(Suppl 16):10-17.

46. Ryan KA, Vederman AC, McFadden EM, et al. Differential executive functioning performance by phase of bipolar disorder. Bipolar Disord. 2012;14:527-536.

47. Ryan KA, Vederman AC, Kamali M, et al. Emotion perception and executive functioning predict work status in euthymic bipolar disorder. Psychiatry Res. 2013;210:472-478.

48. Marshall DF, Walker SJ, Ryan KA, et al. Greater executive and visual memory dysfunction in comorbid bipolar disorder and substance use disorder. Psychiatry Res. 2012;200:252-257.

49. Marshall DF, Passarotti AM, Ryan KA, et al. Deficient inhibitory control as an outcome of childhood trauma. Psychiatry Res. 2016;235:7-12.

50. Stringer D, Marshall D, Pester B, et al. Openness predicts cognitive functioning in bipolar disorder. J Affect Disord. 2014;168:51-57.

51. Delis D, Kaplan E, Kramer J, Ober BA. California Verbal Learning Test-II. San Antonio, TX: The Psychological Corporation; 2000.

52. Rey A. L'examen psychologique dans les cas d'encéphalopathie traumatique. (Les problems.) [The psychological examination in cases of traumatic encepholopathy. Problems.]. Archives de Psychol.1941;28:215-285.

53. Tiffin J, Asher EJ. The purdue pegboard: norms and studies of reliability and validity. J Applied Psychol. 1948;32:234-247.

54. Heaton RK. A Manual for the Wisconsin Card Sorting Test. Odessa, FL: Psychological Assessment Resources; 1981.

55. Golden C. Stroop Color and Word Test. Chicago, IL: Stoelting; 1978.

56. Benton AL, Hamsher KD. Multilingual Aphasia Examination. lowa City, IA: The University of lowa; 1976.

57. Wechsler D. Wechsler Abbreviated Scale of Intelligence (WASI) Manual. San Antonio, TX: The Psychological Corporation; 1999.

58. Lezak MD. Neuropsychological Assessment: New York, NY: Oxford University Press; 2004.

59. Yates AJ. The use of vocabulary in the measurement of intellectual deterioration; a review. J Mental Sci. 1956;102:409-440.

60. Weisenbach SL, Marshall D, Weldon AL, et al. The double burden of age and disease on cognition and quality of life in bipolar disorder. Int J Geriatr Psychiatry. 2014;29:952-961.

61. Bora E, Yucel M, Pantelis C. Cognitive endophenotypes of bipolar disorder: a meta-analysis of neuropsychological deficits in euthymic patients and their first-degree relatives. J Affect Disord. 2009;113:1-20.

62. Martino DJ, Igoa A, Marengo E, Scapola M, Ais ED, Strejilevich SA. Cognitive and motor features in elderly people with bipolar disorder. J Affect Disord. 2008;105:291-295.

63. Langenecker SA, Saunders EFH, Kade AM, Ransom MT, McInnis MG. Intermediate: cognitive phenotypes in bipolar disorder. J Affect Disord. 2010;122:285-293.

\section{SUPPORTING INFORMATION}

Additional Supporting Information may be found online in the supporting information tab for this article.

How to cite this article: Hinrichs $\mathrm{KH}$, Easter RE, Angers K, et al. Influence of cognitive reserve on neuropsychological functioning in bipolar disorder: Findings from a 5-year longitudinal study. Bipolar Disord. 2017;19:50-59. https://doi. org/10.1111/bdi.12470 